\title{
Computation of the Temperature Distribution in Cylindrical High-Pressure Furnaces
}

\author{
Meyer Waxman and John R. Hastings \\ Institute for Basic Standards, National Bureau of Standards, Washington, D.C. 20234
}

(April 22, 1971)

\begin{abstract}
The steady-state temperature distribution in typical cylindrical high-pressure furnaces has been computed from analytic solutions for various boundary conditions. Either the temperature variation along the cylindrical heater or the power dissipation per unit length is prescribed. The results are tabulated and discussed as an aid in the design of high-pressure furnaces and in the estimation of temperature gradients. Topics considered include: (1) the reduction of temperature gradients around the center of the furnace, (2) the effect of temperature irregularities along the heater, and (3) the effect of the relative thermal conductivity of neighboring components.

Key words: Analytic solutions; boundary conditions; computation, temperature distribution; furnaces, high-pressure; high-pressure furnaces; high-temperature, high-pressure research; power dissipation; temperature distribution, steady-state; temperature gradients; temperature irregularities; temperature variation; thermal conductivity.
\end{abstract}

\section{Introduction}

Activity in high-temperature, high-pressure research has greatly increased in recent years. Such work is of both scientific and technological importance and significantly extends our knowledge of the phase diagrams and equations of state of the solids and liquids studied. In the design of experiments and in the detailed interpretation of results, the accuracy and uniformity of the temperature and pressure require careful attention. This paper deals with the computation of the temperature distribution in cylindrical furnaces of the type characteristic of static high-pressure apparatus. Since the pressure does not appear explicitly in the computation, the results are equally applicable to furnaces at any pressure if the geometry and the boundary conditions assumed in this paper are a suitable approximation to the actual physical conditions.

Several problems arise in the maintenance and measurement of high temperatures in high-pressure media. The small size of the pressurized region within a massive metal apparatus restricts the size of the furnace components and thus limits the amount of thermal insulation that separates the sample from the heater and from the surroundings. As a result there may be a large flux of heat across the sample with correspondingly large temperature gradients. Temperature irregularities along the heater may cause additional gradients across the sample. Temperatures in high-pressure furnaces are usually measured with thermocouples. Pressure modifies the temperature-emf relationship of a thermocouple by as much as an estimated 5 to 10 percent under experimental conditions $[1,2] .{ }^{1}$ Because of the temperature and pressure gradi-

1 Figures in brackets indicate the literature references at the end of this paper. ents, the details of positioning the thermocouples influence the readings obtained with them [3].

This paper grew out of our interest in designing a highpressure furnace in which temperature gradients across the sample would be both small and well-known-for

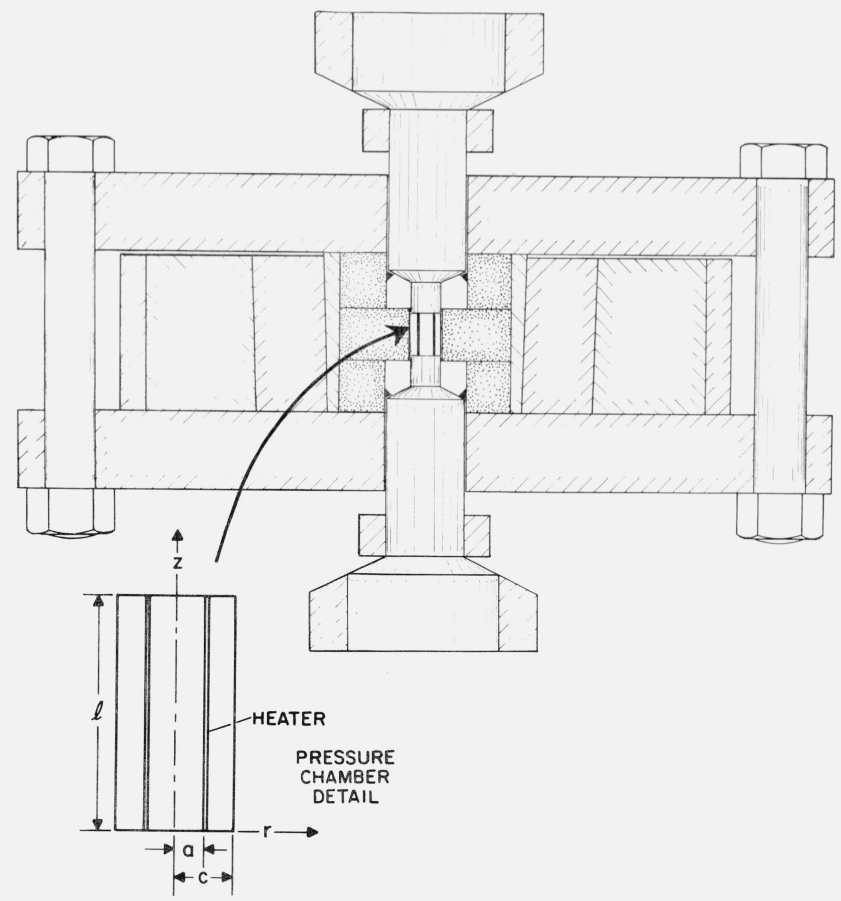

FIgURE 1. Schematic diagram of typical high-pressure furnace with coaxial cylindrical heater. 
example, known to within 0.1 percent of the difference between the highest temperature in the furnace and the temperature of the surrounding metal apparatus. A survey of the literature indicated that the kind of detailed temperature distribution data needed in the design of such a furnace was not available. Because of the various difficulties in the determination of this sort of data by experimental trial and error for different furnace designs, we chose, instead, to compute the temperature distribution for several alternative conditions. Most high-pressure furnace designs can be described by one or a combination of these conditions.

In the present work the steady-state temperature distribution in typical cylindrical high-pressure furnaces has been computed from analytic solutions for various boundary conditions. A schematic diagram of such a typical furnace is shown in figure 1 , in which a cylindrical heater of negligible thickness, radius $a$, and length $l$ is located between opposed pistons in a massive metal apparatus. Either the temperature variation along the cylindrical heater or the power dissipation per unit length is prescribed. Perfect thermal contact is assumed at all boundaries because of the intimate contact created by the high pressure. Angular symmetry and homogeneous, isotropic materials are also assumed throughout this paper.

\section{General Solution for a Prescribed Surface Temperature Variation}

Consider a simple cylinder, $0 \leq r<a, 0<z<l$, with a prescribed temperature $f(z)$ along the cylindrical surface at $r=a$ and a constant temperature, taken as zero for convenience, over the end surfaces. The temperature potential $v(r, z)$ within the cylinder, measured from the temperature at either end, is given by Carslaw and Jaeger $[4]$ as

$$
v(r, z)=\frac{2}{l} \sum_{n=1}^{\infty} \frac{I_{0}(n \pi r / l)}{I_{0}(n \pi a / l)} \sin \frac{n \pi z}{l} \int_{0}^{l} f\left(z^{\prime}\right) \sin \frac{n \pi z}{l} d z^{\prime},
$$

where $\mathrm{I}_{0}(x)$ is the modified Bessel function of the first kind of zero order. We now consider the application of eq (1) for several explicit surface temperature variations.

\subsection{Parabolic Surface Temperature}

For typical furnaces with a smooth temperature variation along the heater from a hot middle to cooler ends, the surface temperature may be approximated by a parabolic function

$$
f(z)=A-(B z-C)^{2} .
$$

By requiring $f(z)$ to be zero at $z=0$ and $z=l$, we obtain $A=C^{2}, B=2 C / l$, and

$$
f(z)=2 B C z-B^{2} z^{2}=\left(4 C^{2} z / l\right)(1-z / l) .
$$

After performing the integration in eq $(1)$, we have

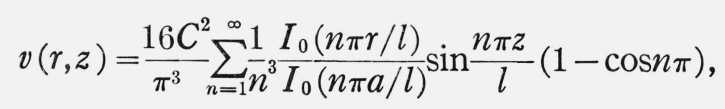

where $C$ is an arbitrary scaling factor. The temperature potential within cylindrical furnaces for the particular geometrical conditions $l=8 a$ and $l=16 a$ is shown in table 1 and table 2 , respectively. Since the furnaces are symmetric about the midplane, $z / l=0.50$, the tables show the potential explicitly for only half the furnace. The computed potential is scaled so that its value in the

\begin{tabular}{|c|c|c|c|c|c|}
\hline$z / l$ & 0.0 & 0.3 & 0.6 & 0.8 & 1.0 \\
\hline 0.50 & 0.9688 & 0.9716 & 0.9800 & 0.9888 & 1.0000 \\
\hline .45 & .9588 & .9616 & .9700 & .9788 & .9900 \\
\hline .40 & .9288 & .9316 & .9400 & .9488 & .9600 \\
\hline .35 & .8788 & .8816 & .8900 & .8988 & .9100 \\
\hline .30 & .8089 & .8117 & .8201 & .8288 & .8400 \\
\hline .25 & .7190 & .7218 & .7302 & .7388 & .7500 \\
\hline .20 & .6095 & .6122 & .6204 & .6289 & .6400 \\
\hline .15 & .4807 & .4832 & .4911 & .4993 & .5100 \\
\hline .10 & .3338 & .3360 & .3428 & .3501 & .3600 \\
\hline .05 & .1715 & .1729 & .1774 & .1825 & .1900 \\
\hline .00 & .0000 & .0000 & .0000 & .0000 & .0000 \\
\hline
\end{tabular}
midplane at $r / a=1.0$, the middle of the heater, is unity.

TABLE 1. Temperature potential within a cylindrical furnace: parabolic surface temperature, $\mathrm{l}=8 \mathrm{a}$

TABLE 2. Temperature potential within a cylindrical furnace: parabolic surface temperature, $\mathrm{l}=16 \mathrm{a}$

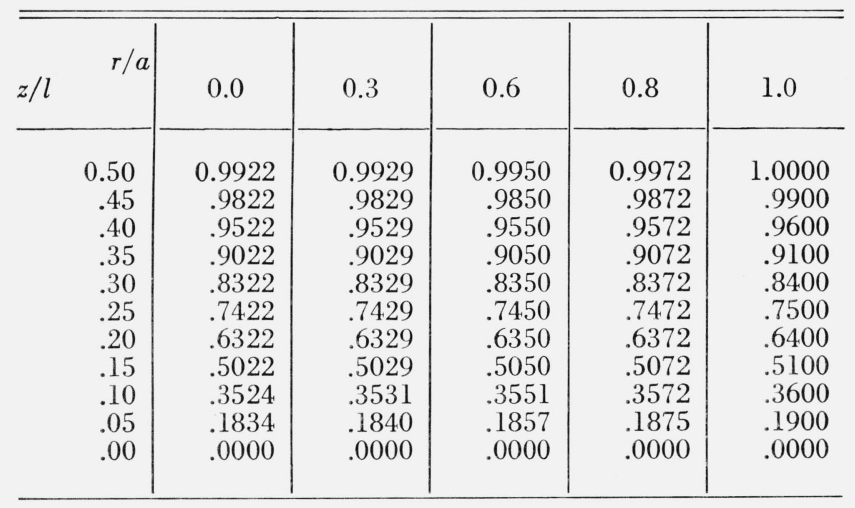

The temperature potential $v(r, z)$ at any point within the cylindrical furnace is very simply related to the actual temperature $T(r, z)$ at that point by the equation

$$
v(r, z)=\frac{T(r, z)-T(r, 0)}{T(a, l / 2)-T(r, 0)},
$$

where $T(r, 0)$ is the reference temperature over the ends of the furnace and $T(a, l / 2)$ is the temperature at the middle of the heater. If, for example, the middle of the heater is $1000{ }^{\circ} \mathrm{C}$ hotter than the ends of the furnace, table 1 shows that the cylindrical midpoint, $r / a=0.0$ and $z / l=0.50$, is $968.8{ }^{\circ} \mathrm{C}$ hotter than the ends of the furnace. If the temperature over the ends of the furnace is $100{ }^{\circ} \mathrm{C}$, the temperature at these other locations becomes $1100{ }^{\circ} \mathrm{C}$ and $1068.8^{\circ} \mathrm{C}$, respectively.

Table 1 shows that the temperature potential in the midplane at $r / a=0.0$, the cylindrical midpoint, is approximately 3 percent less than that at $r / a=1.0$, the middle of the heater, and 2 percent less than that at $r / a=0.8$. In terms of the preceding example, these potential differences represent temperature differences of approximately 
30 and $20^{\circ} \mathrm{C}$. The longitudinal variation from $z / l=0.50$ to 0.45 at any radius is 1 percent. The cylindrical midpoint is a saddle point at which the radial temperature gradient is positive and the longitudinal gradient is negative. For the more elongated furnace of table 2 the potential at the cylindrical midpoint is only 0.8 percent less than that at the middle of the heater and 0.5 percent less than that at $r / a=0.8, z / l=0.50$. The longitudinal variation from $z / l=0.50$ to 0.45 is still 1 percent, of course, although relative to the radius, the corresponding longitudinal displacement is twice as long as in table 1.

\subsection{Generalized Parabolic Surface Temperature With a Constant Central Section}

In practice the small size of the pressurized region restricts the opportunity for elongation of the furnace in order to reduce the temperature gradients around its center. Another way to reduce these gradients is to supply extra heat near the ends of the furnace to compensate for the large losses to the surroundings and thus maintain a substantially constant temperature about the middle of the heater. To represent this section of constant temperature, we generalize the preceding problem by splitting the parabola at its midpoint and inserting a horizontal line segment there. Since the maximum value of $f(z)$ is $C^{2}$, which occurs at $z=C / B$, we obtain

$$
\begin{aligned}
f(z) & =2 B C z-B^{2} z^{2}, 0<z \leqslant C / B, \\
& =C^{2}, C / B \leqslant z \leqslant l-C / B, \\
& =2 B C(l-z)-B^{2}(l-z)^{2}, l-C / B \leqslant z<l .
\end{aligned}
$$

After substituting in eq (1) and performing the integration, we have

$$
\begin{gathered}
v(r, z)=\frac{4 B^{2} l^{2}}{\pi^{3}} \sum_{n=1}^{\infty} \frac{1}{n} n^{3} \frac{I_{0}(n \pi r / l)}{I_{0}(n \pi a / l)} \sin \frac{n \pi z}{l}(1-\cos n \pi) \\
\cdot\left(1-\cos \frac{n \pi C}{B l}\right) .
\end{gathered}
$$

When $C / B=l / 2$, eq (5) reduces to eq (3) and eq (6) reduces to eq (4), since $\cos n_{\pi} / 2$ vanishes for odd $n$ and $1-\cos n_{\pi}$ vanishes for even $n$. The temperature potential within cylindrical furnaces for which $l=8 a$ is shown

\begin{tabular}{|c|c|c|c|c|c|}
\hline$z / l$ & 0.0 & 0.3 & 0.6 & 0.8 & 1.0 \\
\hline 0.50 & 0.9922 & 0.9931 & 0.9957 & 0.9979 & 1.0000 \\
\hline .45 & .9885 & .9898 & .9934 & .9967 & 1.0000 \\
\hline .40 & .9750 & .9773 & .9841 & 9911 & 1.0000 \\
\hline .35 & .9454 & 9487 & .9588 & .9697 & .9844 \\
\hline .30 & .8927 & .8966 & .9085 & 9210 & .9375 \\
\hline .25 & .8125 & .8166 & .8292 & .8423 & .8594 \\
\hline .20 & .7029 & .7071 & .7197 & .7329 & .7500 \\
\hline .15 & .5638 & .5678 & .5799 & .5927 & 6094 \\
\hline .10 & .3966 & .4000 & .4106 & .4221 & .4375 \\
\hline .05 & .2056 & .2077 & .2146 & .2226 & .2344 \\
\hline .00 & .0000 & .0000 & .0000 & .0000 & .0000 \\
\hline
\end{tabular}
in table 3 for $C / B=l / 2.5$ and in table 4 for $C / B=l / 4$.

TABLE 3. Temperature potential within a cylindrical fur. nace: generalized parabolic surface temperature with central fifth at constant temperature, $1=8 \mathrm{a}$
The temperature variations around the cylindrical midpoint are indeed much smaller in these tables than in table 1. For example, the total radial variation in the midplane is 3.1 percent in table $1,0.8$ percent in table 3 ,

\begin{tabular}{|c|c|c|c|c|c|}
\hline$z / l$ & 0.0 & 0.3 & 0.6 & 0.8 & 1.0 \\
\hline 0.50 & 0.9989 & 0.9990 & 0.9994 & 0.9997 & 1.0000 \\
\hline .45 & .9983 & .9985 & .9991 & .9996 & 1.0000 \\
\hline .40 & .9961 & .9966 & .9979 & .9990 & 1.0000 \\
\hline .35 & .9901 & .9913 & .9945 & .9973 & 1.0000 \\
\hline .30 & .9748 & .9776 & .9855 & .9927 & 1.0000 \\
\hline .25 & .9386 & .9441 & .9606 & .9778 & 1.0000 \\
\hline .20 & .8635 & .8715 & .8963 & .9232 & .9600 \\
\hline .15 & .7327 & .7418 & .7697 & .7998 & .8400 \\
\hline .10 & .5388 & .5471 & .5731 & .6015 & .6400 \\
\hline .05 & .2874 & .2928 & .3101 & .3302 & .3600 \\
\hline .00 & .0000 & .0000 & .0000 & .0000 & .0000 \\
\hline
\end{tabular}
and 0.1 percent in table 4 .

TABLE 4. Temperature potential within a cylindrical furnace: generalized parabolic surface temperature with central half at constant temperature, $\mathrm{l}=8 \mathrm{a}$

\subsection{Surface Temperature, a Rectangular Function of Finite Width}

Temperature irregularities exist along real heaters. A detailed model of the irregularities in an actual furnace would greatly increase the complexity of the mathematical analysis; for example, the angular symmetry assumed in the derivation of eq (1) would not generally exist. Rather than neglect such irregularities altogether, we may explore their effect by considering a rectangular function of finite width for the surface temperature

$$
\begin{aligned}
f(z) & =0, & & 0<z<g, \\
& =D, & & g<z<h, \\
& =0, & & h<z<l,
\end{aligned}
$$

where, in general, $g \neq l-h$; that is, the step is not centered at the midplane. After performing the integration in eq (1), we have

$$
\begin{aligned}
v(r, z) & =\frac{2 D}{\pi} \sum_{n=1}^{\infty} \frac{1}{n} \frac{I_{0}(n \pi r / l)}{I_{0}(n \pi a / l)} \sin \frac{n \pi z}{l}\left(\cos \frac{n \pi g}{l}\right. \\
& \left.-\cos \frac{n \pi h}{l}\right) .
\end{aligned}
$$

The temperature potential within a furnace for which $l=8 a$ is shown in tables 5 and 6 for steps of width $0.01 l$ and $0.05 l$ centered at $z / l=0.30$.

The tables show certain features which can be expected as general results of local irregularities in the temperature along the heater. The gradients due to an irregularity modify the gradients otherwise present to an extent that depends upon the width and the height or depth of the irregularity. The radial gradient along a radius leading to a hot spot is increased. The radial gradient elsewhere includes a component due to the hot spot that may be 
negative along the entire radius or positive near the axis and negative near the heater; the latter characteristic is illustrated by the data in the tables for $z / l=0.25$ and 0.35. Longitudinal gradients are also modified. The tables show a slight asymmetry in the gradients above and below the plane of the irregularity as a result of the temperature asymmetry along the heater.

TABLE 5. Temperature potential within a cylindrical furnace: surface temperature, a rectangular function of finite width $(\mathrm{f}(\mathrm{z})=0$ for $0<\mathrm{z}<\mathrm{g}$ and $\mathrm{h}<\mathrm{z}<\mathrm{l}$, $\mathrm{f}(\mathrm{z})=\mathrm{D}$ for $\mathrm{g}<\mathrm{z}<\mathrm{h}, \mathrm{g}=0.295 \mathrm{l}, \mathrm{h}=0.305 \mathrm{l})$, $\mathrm{l}=8 \mathrm{a}$

\begin{tabular}{|c|c|c|c|c|c|}
\hline$z / l$ & 0.0 & 0.3 & 0.6 & 0.8 & 1.0 \\
\hline 1.00 & 0.0000 & 0.0000 & 0.0000 & 0.0000 & 0.0000 \\
\hline .95 & .0000 & .0000 & .0000 & .0000 & .0000 \\
\hline .90 & .0000 & .0000 & .0000 & .0000 & .0000 \\
\hline .85 & .0000 & .0000 & .0000 & .0000 & .0000 \\
\hline .80 & .0000 & .0000 & .0000 & .0000 & .0000 \\
\hline .75 & .0000 & .0000 & .0000 & .0000 & .0000 \\
\hline .70 & .0001 & .0001 & .0000 & .0000 & .0000 \\
\hline .65 & .0002 & .0002 & .0001 & .0000 & .0000 \\
\hline .60 & .0005 & .0004 & .0003 & .0001 & .0000 \\
\hline .55 & .0013 & .0011 & .0007 & .0003 & .0000 \\
\hline .50 & .0033 & .0029 & .0018 & .0009 & .0000 \\
\hline .45 & .0083 & .0074 & .0048 & .0024 & .0000 \\
\hline .40 & .0199 & .0185 & .0132 & .0071 & .0000 \\
\hline .35 & .0400 & .0405 & .0391 & .0280 & .0000 \\
\hline .30 & .0530 & .0579 & .0810 & .1408 & 1.0000 \\
\hline .25 & .0400 & .0405 & .0391 & .0280 & 0.0000 \\
\hline .20 & .0199 & .0185 & .0132 & .0071 & .0000 \\
\hline .15 & .0083 & .0074 & .0048 & .0024 & .0000 \\
\hline .10 & .0032 & .0028 & .0018 & .0009 & .0000 \\
\hline .05 & .0011 & .0009 & .0006 & .0003 & .0000 \\
\hline .00 & .0000 & .0000 & .0000 & .0000 & .0000 \\
\hline
\end{tabular}

TABLE 6. Temperature potential within a cylindrical furnace: surface temperature, a rectangular function of finite width $(\mathrm{f}(\mathrm{z})=0$ for $0<\mathrm{z}<\mathrm{g}$ and $\mathrm{h}<\mathrm{z}<\mathrm{l}$, $\mathrm{f}(\mathrm{z})=\mathrm{D}$ for $\mathrm{g}<\mathrm{z}<\mathrm{h}, \mathrm{g}=0.275 \mathrm{l}, \mathrm{h}=0.325 \mathrm{l})$, $\mathrm{l}=8 \mathrm{a}$

\begin{tabular}{|c|c|c|c|c|c|}
\hline$z / l$ & 0.0 & 0.3 & 0.6 & 0.8 & 1.0 \\
\hline 1.00 & 0.0000 & 0.0000 & 0.0000 & 0.0000 & 0.0000 \\
\hline .95 & .0000 & .0000 & .0000 & .0000 & .0000 \\
\hline .90 & .0000 & .0000 & .0000 & .0000 & .0000 \\
\hline .85 & .0000 & .0000 & .0000 & .0000 & .0000 \\
\hline .80 & .0001 & .0000 & .0000 & .0000 & .0000 \\
\hline .75 & .0001 & .0001 & .0001 & .0000 & .0000 \\
\hline .70 & .0004 & .0003 & .0002 & .0001 & .0000 \\
\hline .65 & .0010 & .0008 & .0005 & .0003 & .0000 \\
\hline .60 & .0025 & .0022 & .0014 & .0007 & .0000 \\
\hline .55 & .0065 & .0057 & .0036 & .0018 & .0000 \\
\hline .50 & .0169 & .0148 & .0094 & .0046 & .0000 \\
\hline .45 & .0428 & .0382 & .0249 & .0126 & .0000 \\
\hline .40 & .1017 & .0948 & .0692 & .0379 & .0000 \\
\hline .35 & .1987 & .2026 & .2029 & .1620 & .0000 \\
\hline .30 & .2588 & .2807 & .3757 & .5599 & 1.0000 \\
\hline .25 & .1987 & .2026 & .2029 & .1620 & 0.0000 \\
\hline .20 & .1016 & .0947 & .0691 & .0379 & .0000 \\
\hline .15 & .0427 & .0381 & .0249 & .0126 & .0000 \\
\hline .10 & .0165 & .0145 & .0092 & .0045 & .0000 \\
\hline .05 & .0055 & .0049 & .0030 & .0015 & .0000 \\
\hline .00 & .0000 & .0000 & .0000 & .0000 & .0000 \\
\hline
\end{tabular}

\section{Solution for Uniform Heating Along the Full Length of the Heater}

In the problems considered thus far, we have prescribed the surface temperature variation along the cylindrical heater. We now prescribe a particular power dissipation along it. Consider a special form of a general problem treated by Laubitz [5] . A composite cylinder of length $l$ and radius $c$, shown in figure 1 , contains a coaxial cylindrical heater of the same length, negligible thickness, and radius $a$, whose power dissipation per unit length $Q$ is independent of time and position; the thermal conductivity is $k_{1}$ for $0 \leq r<a$ and $k_{2}$ for $a<r<c$, and the boundary conditions are $v(c, z)=v(r, l)=v(r, 0)=0$. The distinction between the conductivities, which are assumed to be independent of temperature and pressure, arises because the temperature along the heater is not prescribed. The boundary conditions express the physical fact that the apparatus surrounding the furnace acts as a heat sink.

For the region inside the heater, $0 \leq r<a, 0<z<l$, the temperature potential is

$$
\begin{aligned}
v(r, z) & =\frac{2 Q l}{\pi^{3} k_{2} a} \sum_{n=1}^{\infty} \frac{1}{n^{2}} \\
\cdot & {\left[\frac{(l / n \pi a) I_{0}(n \pi c / l)}{I_{0}(n \pi c / l) K_{0}(n \pi a / l)-K_{0}(n \pi c / l) I_{0}(n \pi a / l)}\right.} \\
+ & \left.\left(\frac{k_{1}}{k_{2}}-1\right) I_{1}(n \pi a / l)\right]^{-1} I_{0}\left(\frac{n \pi r}{l}\right) \sin ^{2}\left(\frac{n \pi}{2}\right) \\
& \cdot \sin \left(\frac{n \pi z}{l}\right),
\end{aligned}
$$

where $I_{0}(x)$ and $I_{1}(x)$ are the modified Bessel functions of the first kind and of zero and first order and $K_{0}(x)$ is the modified Bessel function of the second kind of zero order. If $k_{1}=k_{2}$, eq (9) reduces to

$$
\begin{aligned}
v(r, z)= & \frac{2 Q}{\pi^{2} k_{2}} \sum_{n=1}^{\infty} \frac{1}{n}\left[K_{0}\left(\frac{n \pi a}{l}\right)-K_{0}\left(\frac{n \pi c}{l}\right)\right] \frac{I_{0}(n \pi a / l)}{I_{0}(n \pi c / l)} \\
& \cdot I_{0}(n \pi r / l) \sin ^{2}(n \pi / 2) \sin (n \pi z / l) .
\end{aligned}
$$

The temperature potential inside the heater of a furnace for which $l=4 c=8 a$ is shown in tables 7 through 9 for $k_{1} / k_{2}=0.1,1$, and 10 . As in the earlier tables, the potential is scaled to a maximum value of unity.

Three observations may be made regarding the tables. Near the midplane the temperature along the heater is quite uniform for $k_{1} / k_{2}<1$ and becomes more nearly parabolic as $k_{1} / k_{2}$ increases. To be more specific, for $r / a=1.0$ the potential at $z / l=0.40$ is 0.2 percent less than that at $z / l=0.50$ for $k_{1} / k_{2}=0.1,2.8$ percent less for $k_{1} / k_{2}=10$, and 4 percent less for the parabolic surface temperature of table 1. In practice an experimental sample is commonly located in the vicinity of the axis and the midplane - for example, within the cylindrical volume bounded by $z / l=0.40$ and 0.60 and $r / a=0.6$. Within this volume the maximum potential difference is 0.5 percent of the reference value of unity for $k_{1} / k_{2}=0.1,3.8$ percent for $k_{1} / k_{2}=10$, and 5.1 percent for the parabolic surface temperature of table 1. For constant $Q$ and $k_{2}$ an increase 
in $k_{1}$ causes a decrease in the temperature for $r<a$. This result follows directly from eq (9) and is related physically to the greater flux of heat to the anvils. It is not apparent in the tables because of the scaling.

TABLE 7. Temperature potential within a cylindrical furnace: uniform heating along the full length of the heater of radius a, $\mathrm{l}=4 \mathrm{c}=8 \mathrm{a}$, (thermal conductivity for $0 \leqslant \mathrm{r}<\mathrm{a})=0.1$ (thermal conductivity for $\mathrm{a}<\mathrm{r}<\mathrm{c}$ )

\begin{tabular}{|c|c|c|c|c|c|}
\hline$z / l$ & 0.0 & 0.3 & 0.6 & 0.8 & 1.0 \\
\hline 0.50 & 0.9975 & 0.9978 & 0.9985 & 0.9992 & 1.0000 \\
\hline .45 & .9965 & .9968 & .9978 & .9986 & .9996 \\
\hline .40 & .9931 & .9937 & .9951 & .9965 & .9981 \\
\hline .35 & .9858 & .9868 & .9895 & .9920 & .9950 \\
\hline .30 & .9714 & .9732 & .9783 & .9830 & .9886 \\
\hline .25 & .9439 & .9472 & .9565 & .9655 & .9760 \\
\hline .20 & .8920 & .8979 & .9150 & .9316 & .9512 \\
\hline .15 & .7967 & .8068 & .8365 & .8662 & .9022 \\
\hline .10 & .6290 & .6439 & .6902 & .7400 & .8042 \\
\hline .05 & .3596 & .3740 & .4249 & .4919 & .5985 \\
\hline .00 & .0000 & .0000 & .0000 & .0000 & .0000 \\
\hline
\end{tabular}

TABLE 8. Temperature potential within a cylindrical furnace: uniform heating along the full length of the heater of radius $\mathrm{a}, \mathrm{l}=4 \mathrm{c}=8 \mathrm{a}$, (thermal conductivity for $0 \leqslant \mathrm{r}<\mathrm{a})=($ thermal conductivity for $\mathrm{a}<\mathrm{r}<\mathrm{c})$

\begin{tabular}{|c|c|c|c|c|c|}
\hline$z / l$ & 0.0 & 0.3 & 0.6 & 0.8 & 1.0 \\
\hline 0.50 & 0.9932 & 0.9939 & 0.9958 & 0.9977 & 1.0000 \\
\hline .45 & .9908 & .9916 & .9937 & .9958 & .9984 \\
\hline .40 & .9830 & .9840 & .9869 & .9897 & .9931 \\
\hline .35 & .9680 & .9695 & .9737 & .9779 & .9830 \\
\hline .30 & .9423 & .9445 & .9512 & .9577 & .9657 \\
\hline .25 & .8999 & .9035 & .9140 & .9243 & 9370 \\
\hline .20 & .8314 & .8370 & .8535 & .8699 & .8900 \\
\hline .15 & .7225 & .7309 & .7559 & .7815 & .8133 \\
\hline .10 & .5538 & .5649 & .5998 & .6375 & 6869 \\
\hline .05 & .3087 & .3188 & .3539 & .3994 & .4713 \\
\hline .00 & .0000 & .0000 & .0000 & .0000 & .0000 \\
\hline
\end{tabular}

TABLE 9. Temperature potential within a cylindrical furnace: uniform heating along the full length of the heater of radius a, $\mathrm{l}=4 \mathrm{c}=8 \mathrm{a}$, (thermal conductivity for $0 \leq \mathrm{r}<\mathrm{a})=10$ (thermal conductivity for $\mathrm{a}<\mathrm{r}<\mathrm{c}$ )

\begin{tabular}{|c|c|c|c|c|c|}
\hline$z / l$ & 0.0 & 0.3 & 0.6 & 0.8 & 1.0 \\
\hline 0.50 & 0.9776 & 0.9796 & 0.9857 & 0.9920 & 1.0000 \\
\hline .45 & .9702 & .9723 & .9786 & .9850 & .9931 \\
\hline .40 & .9480 & .9502 & .9568 & .9636 & .9723 \\
\hline .35 & .9098 & .9122 & .9195 & .9270 & .9366 \\
\hline .30 & .8541 & .8569 & .8652 & .8737 & .8845 \\
\hline .25 & .7786 & .7819 & .7914 & .8013 & .8138 \\
\hline .20 & .6803 & .6840 & .6952 & .7068 & .7216 \\
\hline .15 & .5551 & .5595 & .5726 & .5863 & .6039 \\
\hline .10 & .3993 & .4040 & .4185 & .4342 & .4551 \\
\hline .05 & .2114 & .2150 & .2273 & .2426 & .2662 \\
\hline .00 & .0000 & .0000 & .0000 & .0000 & .0000 \\
\hline
\end{tabular}

Further calculations have been performed to study the effects of varying the furnace parameters. The results, which are not shown in the tables, display considerable complexity. For example, for $l=4 c$ the changes in the radial and longitudinal temperature gradients in the vicinity of the axis and the midplane that are caused by a decrease in $a / c$ from 0.50 to 0.25 depend in sign as well as in magnitude on the value of $k_{1} / k_{2}$. Consider the cylindrical volume bounded by $z / l=0.45$ and 0.55 and $r / c=0.25$. Within this volume the maximum potential difference increases slightly from 0.14 percent to 0.18 percent of the reference value of unity as $a / c$ decreases from 0.50 to 0.25 for $k_{1} / k_{2}=0.01$. For $k_{1} / k_{2}=0.1$ the increase is from 0.17 percent to 0.19 percent. For $k_{1} / k_{2}=1$ the maximum potential difference decreases from 0.43 percent to 0.29 percent as $a / c$ decreases from 0.50 to 0.25 . For $k_{1} / k_{2}=10$ the decrease is from 1.30 percent to 0.99 percent, and for $k_{1} / k_{2}=100$ it is from 1.69 percent to 1.65 percent. For $k_{1} / k_{2}=1000$ the maximum potential difference is barely influenced by the decrease in $a / c$, changing from 1.75 percent to 1.76 percent.

\section{Comments}

Although the results shown in the tables are based upon several simplifying assumptions, we believe that they are sufficiently general to be helpful in the design of highpressure furnaces and in the estimation of temperature gradients. By superposition of these results the results of more complicated problems may be obtained. This is the reason for the inclusion of the problem in which temperature irregularities along the heater are approximated by the assumption that the surface temperature is a rectangular function of finite width.

Reported furnace designs have not always illustrated an optimum choice of environmental materials so far as the thermal conductivities are concerned. The tables show that the temperature gradients in the sample region are significantly affected by the relative thermal conductivity of neighboring components as well as by the temperature variation along the heater. In many cases temperature irregularities along the heater may impose a lower limit to the magnitude of the temperature gradients in the sample region.

It is a pleasure to thank Bradley A. Peavy, Jr. for several helpful discussions.

\section{References}

[1] Bundy, F. P., Effect of pressure on emf of thermocouples, J. Appl. Phys. 32, 483-488 (1961).

[2] Hanneman, R. E., and Strong, H. M., Pressure dependence of the emf of thermocouples to $1300^{\circ} \mathrm{C}$ and $50 \mathrm{kbar}, \mathrm{J}$. Appl. Phys. 36, 523-528 (1965).

[3] Hanneman, R. E., and Strong, H. M., Pressure dependence of the emf of thermocouples, J. Appl. Phys. 37, 612-614 (1966).

[4] Carslaw, H. S., and Jaeger, J. C., Conduction of Heat in Solids, 2nd ed. (Clarendon Press, Oxford, 1959), p. 220.

[5] Laubitz, M. J., Mathematical treatment of a four-component furnace, Can. J. Phys. 39, 951-955 (1961). 